The following paper posted here is not the official IEEE published version. The final published version of this paper can be found in the Proceedings of the IEEE International Geoscience and Remote Sensing Symposium, 20-24 September, 2004:pp.1248-1251

Copyright @ 2004 IEEE.

Personal use of this material is permitted. However, permission to reprint/republish this material for advertising or promotional purposes or for creating new collective works for resale or redistribution to servers or lists, or to reuse any copyrighted component of this work in other works must be obtained from the IEEE. 


\section{3-D Broadband Ground-based Polarimetric SAR Data Processing for the Monitoring of Vegetation Growth Variations}

\author{
Zheng-Shu Zhou, Tadashi Hamasaki, Motoyuki Sato \\ Center for Northeast Asian Studies \\ Tohoku University \\ Sendai, Japan \\ \{zszhou, hamasaki, sato\}@cneas.tohoku.ac.jp
}

\author{
Wolfgang-Martin Boerner \\ ECE-CSN \\ University of Illinois at Chicago \\ Chicago, USA \\ boerner@ece.uic.edu
}

\begin{abstract}
SAR is usually used for airborne or space borne remote sensing. It can also advantageously be exploited in a ground-based radar imaging system named Ground-based SAR (GB-SAR). We extended earlier approaches and developed an ultra-wideband, ground-based, fully polarimetric SAR (Pol-GBSAR) system for the monitoring of vegetation growth variations. Measurements on three type trees in different conditions were carried out by the developed SAR system. We proposed effective three-dimensional (3-D) broadband Pol-GB-SAR data processing algorithms in the paper. In situ polarimetric calibration obviously improved the features of the system. 3-D images were reconstructed from the acquired data by a series of signal processing procedures based on a variety of wave equation migration methods. By implementing methods of radar polarimetry, the broadband GB-SAR system has possibility for monitoring changes in tree structure characteristics due to seasonal variations. Interpreted results demonstrated the target scattering characteristics in different vegetation growth situations showed good agreement with the ground truth.
\end{abstract}

Keywords-Ground-based SAR (GB-SAR); Radar Polarimetry; Polarimetric Calibration; 3-D Imaging Algorithms; Short Time Fourier Transform (STFT); Scattering Matrix; Vegetation Variation Identification

\section{INTRODUCTION}

An important feature of electromagnetic wave scattering is its state of polarization, and a wide range of classification algorithms and inversion techniques have recently been developed based on the transformation of polarization state by scattering objects [1-3]. There are three primary ways in which multi-parameter radar measurements can be made: multifrequency, single or multi-baseline interferometry and multipolarization, and more recently a combination of these three approaches. Therefore, the combination of spatial information, spectral information and polarization information with multifrequency and broadband sensors defines the recent advances accomplished in polarimetric SAR systems development [4, 5]. GB-SAR is a new application of the conventional SAR expansion in the spatial domain.

Based on derived GB-SAR principles and simulation results, we extended those earlier approaches and developed an ultra-wideband, ground-based, fully polarimetric SAR system for transverse and oblique vegetation monitoring [6]. The radar system consists of a vector network analyzer, a diagonal dual polarized broadband horn antenna an antenna positioning unit, and a PC-based control unit.

We used the developed SAR system to carry out noninvasive measurements for three types of trees in three different seasons, namely in spring, summer and autumn, respectively. Three-dimensional (3-D) polarimetric images are reconstructed from the acquired data by a series of signal processing procedures based on a variety of wave front reconstruction methods. Combined signal processing methods for broadband ground-based polarimetric SAR data and data interpretations for tree growth monitoring are addressed in this paper.

\section{FIELD EXPERIMENTS FOR TREE GROWTH MONITORING}

Due to the fact that trees are some of the most important factors of the terrestrial vegetation cover, we selected an experimental site with different types of trees used for the noninvasive remote monitoring measurements. The main targets are denoted by $\mathrm{T} 1, \mathrm{~T} 2$ and $\mathrm{T} 3$, and represented three different kinds of trees. T1 is a Japanese Zelkova, which is a deciduous tree that has no leaf in spring, exuberant broad leaves during early summer up to mid-autumn, after then leaves fallen off. $\mathrm{T} 2$ is a Japanese Cedar, which is an evergreen with needles. It almost does not change from spring to winter. T3 is a kind shrub of the Azalea genus which is a short bush surrounded by some plants: Japanese Honeysuckle. From spring to summer, they have very dense foliage while there are some stems and branches after autumn.

We have carried out three measurements at the same exact position for the three different trees in late spring (April 19, 2002), in early summer (May 28, 2002) and in late autumn (November 11, 2002), respectively. There were a few fresh leaves during the first measurement in spring. Very significant growth in leaves and branches was observed in the second measurement in summer, while the third measurement was at a time when the leaves had fallen off. These measurement scenes are shown in Fig. 1 (on page 3 of this paper). HH, VH and VV polarization frequency domain data were acquired which cover frequency range from $1 \mathrm{GHz}$ to $5 \mathrm{GHz}$.

A part of this work was supported by the Grant-In-Aid for Scientific Research (S)14102024 of JSPS. 


\section{3-D IMAGING ALGORITHMS}

\section{A. Signal Processing}

For the acquired broadband Pol-GB-SAR data, a series of different signal processing methods are applied. Due to different data structure, GB-SAR signal processing is not the same as the conventional SAR data processing algorithms. First, the acquired frequency domain data are filtered by the broadband-pass filter; then transformed to the time domain by $I F F T$. By selecting the interesting range at time axis, time gating is employed to the time domain data; then backtransformed to the frequency domain by FFT. The frequency domain data are calibrated by radar system calibration coefficients. Thereafter, the calibrated data are compressed by matched filtering, in which the reference signal was the reflection from an aluminum plate measured by the same radar system and parameters. After using the IFFT again, the time domain data are obtained. Finally, 3-D wave equation-based diffraction stacking migration is applied to focus the time domain scattering signal on targets in the spatial domain; and the special domain data sets are obtained. More detailed can be referred to [6].

\section{B. Time Gating}

Time gating is a very useful spatial filtering process for reducing antenna direct coupling and noise beyond the region of interest. It uses a windowing function to filter a time domain signal and picks up the signal of interest only. The basic formulation of time gating is shown in (1).

$$
f(t)=f_{0}(t) \cdot w(\zeta)
$$

$f_{0}(t)$ is a original time domain signal, and $w(\zeta)$ is a windowing function. $f(t)$ is the gated signal.

\section{In Situ Polarimetric Calibration}

A modified polarimetric Radar Cross Section (RCS) calibration technique using two orientations of the dihedral corner reflectors as calibration targets is introduced [6, 7]. From the theoretical value of two calibrators and measured data, we can recover calibration coefficients. With the use of the calibration coefficients, the auto-calibrated results are presented in Table 1 resulting in observable improvements after calibration, especially in the phase terms. Although neglecting effects of noise in the calibration measurement, the improvements due to calibration have been achieved.

TABLE 1. AUTO-CALIBRATED SCATTERING MATRICES

\begin{tabular}{|c|l|l|}
\hline & Measured scattering matrix & Calibrated scattering matrix \\
\hline $0^{\circ}$ dihedral & {$\left[\begin{array}{ll}0.9709 e^{-j 1148^{\circ}} & 0.0173 e^{-j 1156^{\circ}} \\
0.0166 e^{-j 1064^{\circ}} & 1\end{array}\right]$} & {$\left[\begin{array}{ll}1.0004 e^{-j 180.0^{\circ}} & 0.0186 e^{-j 109.0^{\circ}} \\
0.0182 e^{-j 116.6^{\circ}} & 1\end{array}\right]$} \\
\hline $45^{\circ}$ dihedral & {$\left[\begin{array}{ll}0.0850 e^{-j 91.1^{\circ}} & 1 \\
1.0095 e^{j 1.4^{\circ}} & 0.0175 e^{-j 1667^{\circ}}\end{array}\right]$} & {$\left[\begin{array}{lll}0.0001 e^{j 14.45^{\circ}} & 1 \\
1 & 0\end{array}\right]$} \\
\hline
\end{tabular}

\section{3-D Wave Equation-based Migration and Spatial Extrapolation}

We introduce a modified wave equation-based time-space domain SAR algorithms for generalized 3-D SAR imaging. A 3-D SAR image can be reconstructed by using the data set acquired with the 2-D aperture by implementing the wave equation-based migration method. Wave equation-based migrations are often used for image reconstruction by wave field extrapolation, which is similar yet quite distinct from that of microwave holography. The diffraction stacking is an integral solution of the wave equation. It can extrapolate wave fields of an observation aperture to larger 3-D space. The 3-D image reconstruction via modified diffraction stacking, derived from a back-projection algorithm $[8,9]$, is obtained in terms of time delays by

$$
\begin{gathered}
P\left(x_{i}, y_{j}, z_{k}\right)=\iint_{S} f\left(t_{i j k}, x_{r}, z_{r}\right) \cdot A(\theta) d z_{r} d x_{r} \\
\begin{aligned}
t_{i j k}\left(x_{r}, z_{r}\right)= & \left(\sqrt{\left(x_{t}-x_{i}\right)^{2}+y_{j}^{2}+\left(z_{t}-z_{k}\right)^{2}}\right. \\
+ & \left.\sqrt{\left(x_{r}-x_{i}\right)^{2}+y_{j}^{2}+\left(z_{r}-z_{k}\right)^{2}}\right) / v
\end{aligned}
\end{gathered}
$$

which is the round-trip delay of the echoed signal for the target at $\left(x_{i}, y_{j}, z_{k}\right)$ when the receiving antenna is at $\left(x_{r}, 0, z_{r}\right)$, and $S$ is the 2-D synthetic aperture, $A(\theta)$ is a antenna directivity compensation function. This migration algorithm focuses the time domain scattering signal on the target in space while antenna radiation directivity compensation is done synchronously and simultaneously by computing (2).

\section{E. Spatial-Frequency Transform}

The short time Fourier transform (STFT) is a compromise between the time and the frequency view of a signal, and is thereby better suited for analysis of non-stationary signals than the ordinary Fourier Transform is. STFT computes a timefrequency distribution of an input signal $\mathrm{x}$ within a given frequency interval as a sequence of short-time spectra of windowed signal segments with constant length. The STFT is expressed as [10]:

$$
X(f, \tau)=\int_{-\infty}^{\infty} x(t) \gamma(t-\tau) e^{-j 2 \pi f t} d t
$$

One of the disadvantages of the STFT is that all frequencies of the signal are analyzed using the same resolution. If we want to obtain precise frequency domain resolution and do not care about spatial resolution in range direction, we can use a long time window to cover the one spatial dimension entirely or do not use the time window to transform spatial domain data to wave-number domain by Fourier transformation. We have the following transform relation.

$$
p(z, x, y) \Rightarrow P\left(z, x, k_{y}\right)
$$

where $p(z, x, y)$ is the 3 -D spatial domain data and $P\left(z, x, k_{y}\right)$ is the spatial-wavenumber domain data. And $k_{y}=\frac{2 \pi}{\lambda_{y}}=\frac{2 \pi f}{c}$, where $f$ is the frequency and $\mathrm{c}$ is the speed of light, 
$f=\frac{c \cdot k_{y}}{2 \pi}$. Therefore, we can obtain frequency information from 3-D spatial domain data.

\section{DATA INTERPRETATIONS FOR TREE GROWTH VARIATIONS}

\section{A. Broadband Polarimetric Images}

Applying the migrated dataset, vertical polarimetric images of three measurements at range $12.9 \mathrm{~m}, 9.8 \mathrm{~m}$ and $8.3 \mathrm{~m}$ are shown in Figs. 2, 3 and 4. Red color indicates the $\mathrm{HH}$ polarization. Green color indicates the VH polarization and blue shows the VV component. Red color shows the reflection from trunks, some horizontal branches and ground clutter. Green color implies backscattering from leaves, some slant branches and ground clutter. Blue color illustrates the reflections from vertical trunks and branches.

From the images of Figs. 2(a), 2(b) and 2(c), the different phenomena can be observed. The strong reflection (red, blue) in the left lower part shows scattering from the trunk of tree T1. On the upper part, the blue shows the vertical branch. The reflections at two sides of trunk of the tree $\mathrm{T} 1$ indicate the branches. Obviously, the green part in Fig. 2(b) is stronger than others due to the many leaves and the growing branches of tree $\mathrm{T} 1$ in summer. We can find the corresponding components indicated by marks in Fig. 1. In Fig. 3, the strong reflection (red, blue and white color) in the center shows scattering from the trunk of tree T2. The upper part indicates the leaves of tree T2. They are almost same due to the evergreen of tree T2. Because of many plants around the tree T3 in summer, the large area of reflection appeared at the corresponding position in Fig. 4(b). There are stronger reflection in the summer images due to the dense branches and leaves. In Fig. 4, front

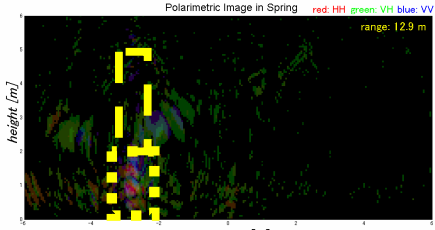

(a)

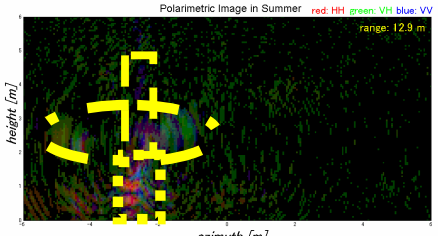

(b)

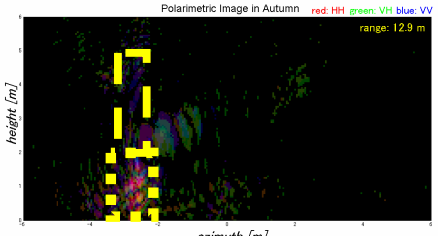

(c)

Figure 2. Broadband vertical profiles of $\mathrm{T} 1$ at range of $12.9 \mathrm{~m}$ in: (a) spring, (b) summer and (c) autumn.

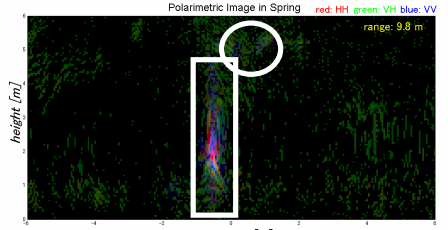

(a)

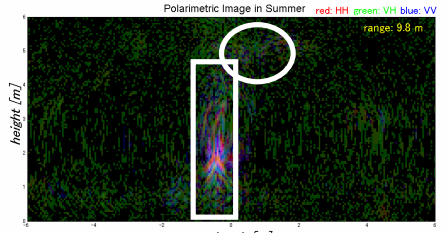

(b)

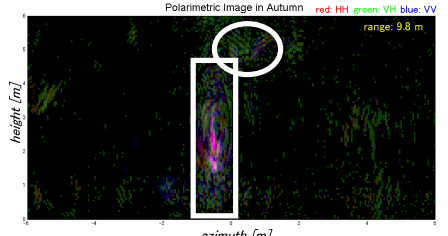

(c)

Figure 3. Broadband vertical profiles of $\mathrm{T} 2$ at range of $9.8 \mathrm{~m}$ in: (a) spring, (b) summer and (c) autumn. part leaves and branches of tree $\mathrm{T} 2$ and difference of tree $\mathrm{T} 3 \mathrm{in}$ variant season can be observed. Hence, we can assume that the main reason is volume scattering due to different volumes with variant seasons. Although the shapes of trees were very similar, the water content of trees in spring was higher than ones in autumn. That caused different reflectivity of targets.

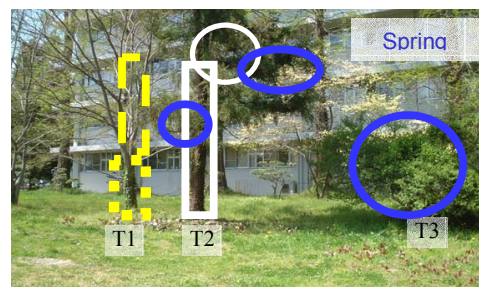

(a)

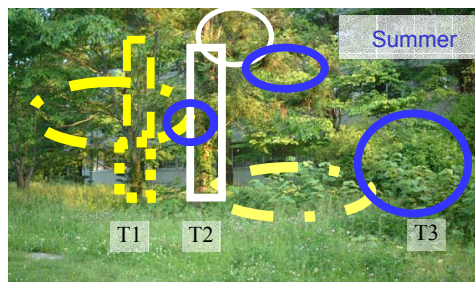

(b)

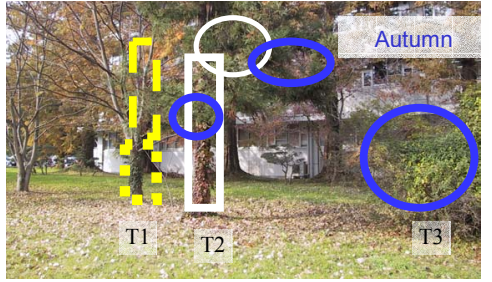

(c)

Figure 1. Test scenes of different trees in: (a) spring, (b) summer and (c) autumn.

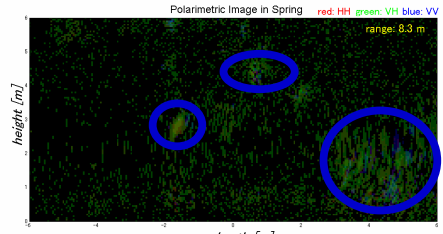

(a)

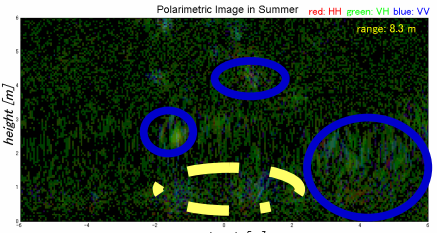

(b)

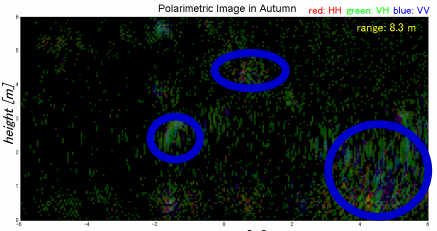

(c)

Figure 4. Broadband vertical profiles of $\mathrm{T} 3$ at range of $8.3 \mathrm{~m}$ in: (a) spring, (b) summer and (c) autumn. 
TABLE 2. SCATTERING MATRICES AT $3 \mathrm{GHz}$

\begin{tabular}{|c|cc|c|c|}
\hline & A: Tree 1 & B: Tree 2 & C: Tree 3 \\
\hline \multirow{2}{*}{ Spring } & {$\left[\begin{array}{lll}e^{j 55.93^{\circ}} & 0.0199 e^{-j 117.58^{\circ}} \\
& 0.0199 e^{-j 117.58^{\circ}} & 0.5229 e^{-j 56.58^{\circ}}\end{array}\right]$} & {$\left[\begin{array}{ll}e^{-j 146.31^{\circ}} & 0.6393 e^{j 34.42^{\circ}} \\
0.6393 e^{j 34.42^{\circ}} & 0.7574 e^{j 35.45^{\circ}}\end{array}\right]$} & {$\left[\begin{array}{ll}e^{-j 152.92^{\circ}} & 1.4826 e^{-j 153.19^{\circ}} \\
1.4826 e^{-j 153.19^{\circ}} & 1.0974 e^{-j 150.39^{\circ}}\end{array}\right]$} \\
\hline \multirow{2}{*}{ Summer } & {$\left[\begin{array}{lll}e^{j 52.24^{\circ}} & 0.4803 e^{-j 123.23^{\circ}} \\
0.4803 e^{-j 123.23^{\circ}} & 1.5813 e^{j 54.29^{\circ}}\end{array}\right]$} & {$\left[\begin{array}{lll}e^{j 34.67^{\circ}} & 0.4718 e^{j 34.36^{\circ}} \\
0.4718 e^{j 34.36^{\circ}} & 1.3186 e^{j 34.40^{\circ}}\end{array}\right]$} & {$\left[\begin{array}{ll}e^{j 26.49^{\circ}} & 1.1656 e^{-j 155.75^{\circ}} \\
1.1656 e^{-j 155.75^{\circ}} & 1.0230 e^{j 25.21^{\circ}}\end{array}\right]$} \\
\hline \multirow{2}{*}{ Autumn } & {$\left[\begin{array}{lll}e^{j 122.77^{\circ}} & 0.1329 e^{j 49.63^{\circ}} \\
0.1329 e^{j 49.63^{\circ}} & 1.945 e^{j 54.11^{\circ}}\end{array}\right]$} & {$\left[\begin{array}{lll}e^{j 34.86^{\circ}} & 0.6198 e^{j 35.47^{\circ}} \\
0.6198 e^{j 35.47^{\circ}} & 1.2708 e^{j 38.56^{\circ}}\end{array}\right]$} & {$\left[\begin{array}{ll}e^{-j 155.0^{\circ}} & 0.4570 e^{-j 152.51^{\circ}} \\
0.4570 e^{-j 152.51^{\circ}} & 1.0769 e^{j 25.88^{\circ}}\end{array}\right]$} \\
\hline
\end{tabular}

\section{B. Identify Tree Growth Variation by Scattering Matrix}

From migrated data, spatial frequency domain data can be created by STFT. Three observation points A, B, C from main targets area were selected, which correspond to T1, T2 and T3, respectively. At each point, scattering matrix was calculated from their spatial frequency domain data. Scattering matrixes of $3 \mathrm{GHz}$ for each measurement and each target are shown in Table 2 .

For point $\mathrm{A}$, the $\mathrm{HH}$ and $\mathrm{VV}$ are dominated due to horizontal scatterers, ground clutter and vertical branches and the trunk of tree T1. Moreover, values of the cross polarization terms in spring and autumn are smaller than the ones for summer. The fact may be that there were many larger leaves in summer to cause stronger VH reflection. We can also find that the co-polarization terms of point $\mathrm{B}$ are dominant but the $\mathrm{VH}$ returns are almost alike and stronger than those at point $\mathrm{A}$. It is due to the fact that point $B$ is located at an evergreen tree. The conifer needles and branches cause large VH reflection. For point $\mathrm{C}, \mathrm{VH}$ terms are stronger than $\mathrm{HH}$ and $\mathrm{VV}$ terms in spring and in summer due to number leaves and plants. When leaves fall off, the VH term became smaller.

\section{CONCLUSIONS}

Using the developed Pol-GB-SAR system, three measurements for three types of trees in spring, summer and autumn were contiguously produced. In situ polarimetric calibration results showed improvements of the specific polarimetric characteristics for the broadband Pol-GB-SAR system. 3-D spatial domain data were produced from the acquired data by a series of signal processing procedures based on a variety of wave equation migration methods. Combined signal processing methods for broadband Pol-GB-SAR data were addressed, in particular time gating, and wave equationbased migration. They made up for the drawbacks of the ground-based imaging system, for instance, restraining reflection of ground clutter, and extrapolating the imaging space. By implementing methods of radar polarimetry, changes in tree structure characteristics, such as growth of leaves and branches due to seasonal variations, could be identified. Further, spatial-frequency transform is used for obtaining spatial-frequency information from the migrated spatial domain data. Interpreted results demonstrated the target scattering characteristics in different vegetation growth situations showed good agreement with the ground truth. Scattering mechanisms of different components of trees were demonstrated by polarimetric analysis techniques.

The experimental results demonstrated the good polarimetric performance of the developed SAR imaging system, which should find many other applications to be desirable in order to more clearly distinguish the associated scattering mechanisms. It can also be used as ground truth demonstration tool for airborne SAR and space-borne SAR in a great variety of applications.

\section{REFERENCES}

[1] W.-M. Boerner et al, Polarimetry in Remote Sensing: Basic and Applied Concepts, Chapter 5 in Manual of Remote Sensing, 3rd ed., vol. 2, F M Henderson, A J Lewis Ed. New York: Wiley, 1998, pp. 271-358.

[2] S. R. Cloude, and E. Pottier, "A review of target decomposition theorems in radar polarimetry," IEEE Trans. Geosci. Remote Sensing, vol. 34, pp. 498-518, 1996.

[3] J. S. Lee, M. R. Grunes, E. Pottier, L. Ferro-Famil, "Segmentation of polarimetric SAR images that preserves scattering mechanisms," Proc. EUSAR2002, June 2002.

[4] W.-M. Boerner, "Recent advances in extra-wideband polarimetry, interferometry and polarimetric interferometry in synthetic aperture remote sensing, and its applications," IEE Proc. Radar Sonar Navigation, Special Issue of the EUSAR-02, vol. 150, pp. 113-125, 2003.

[5] E. Pottier, S. R. Cloude, and W.-M. Boerner, "Recent development of data processing in polarimetric and interferometric SAR," Radio Science Bulletin, (ed. R.W. Stone), Special Issue on URSI-GA-03; Maastricht; NL, RSB-304, March 2003, pp. 48-59, 2003.

[6] Z.-S. Zhou, Application of a Ground-based Polarimetric SAR System for Environmental Study, Doctoral Dissertation of Tohoku University, Japan, 2003.

[7] J.-R. J. Gau, and W. D. Burnside, "New polarimetric calibration technique using a single calibration dihedral," IEE Proc. Microw. Antennas Propag., vol. 142, pp. 19-25, 1995.

[8] Z.-S. Zhou, W.-M. Boerner and M. Sato, "Development of a groundbased polarimetric broadband SAR system for non-invasive groundtruth validation in vegetation monitoring," IEEE Trans. Geosci. Remote Sensing, in press.

[9] M. Soumekh, Synthetic Aperture Radar Signal Processing, New York: John Wiley \& Sons, 1999, pp. 212-215.

[10] G. F. Boudreaux-Bartels, "Mixed time-frequency signal transformations," Chapter 12 in The Transforms and Applications Handbook, 2nd ed., A. D. Poularikas Ed, Boca Raton, Florida: CRC Press LLC, 2000, pp. 12.6-12.8. 